TITLE:

\title{
Electrophoresis of long deoxyribonucleic acid in curved channels: The effect of channel width on migration dynamics
}

\section{AUTHOR(S):}

Ueda, M; Hayama, T; Takamura, Y; Horiike, Y; Dotera, T; Baba, Y

\section{CITATION:}

Ueda, M ...[et al]. Electrophoresis of long deoxyribonucleic acid in curved channels: The effect of channel width on migration dynamics. JOURNAL OF APPLIED PHYSICS 2004, 96(5): 2937-2944

\section{ISSUE DATE:}

2004-09-01

URL:

http://hdl.handle.net/2433/50198

\section{RIGHT:}

Copyright 2004 American Institute of Physics. This article may be downloaded for personal use only. Any other use requires prior permission of the author and the American Institute of Physics. 


\title{
Electrophoresis of long deoxyribonucleic acid in curved channels: The effect of channel width on migration dynamics
}

\author{
Masanori Ueda ${ }^{\text {a) }}$ \\ Department of Medicinal Chemistry, Faculty of Pharmaceutical Sciences, The University of Tokushima, \\ Tokushima 770-8505, Japan and CREST, Japan Science and Technology Corporation, \\ Chiba 292-0812, Japan \\ Tetsuya Hayama, Yuzuru Takamura, and Yasuhiro Horiike \\ Department of Materials Science, School of Engineering, The University of Tokyo, Tokyo 113-8656, Japan \\ Tomonari Dotera \\ Saitama Study Center, The University of the Air, Saitama 331-0851, Japan \\ Yoshinobu Baba \\ Department of Medicinal Chemistry, Faculty of Pharmaceutical Sciences, The University of Tokushima, \\ Tokushima 770-8505, Japan and CREST, Japan Science and Technology Corporation, \\ Chiba 292-0812, Japan
}

(Received 5 January 2004; accepted 3 June 2004)

\begin{abstract}
We investigated the dynamics of long deoxyribonucleic acid (DNA) migrating through curved channels under electric fields. Long DNA exhibits large conformational changes in the curved channels because of the inhomogeneity of the electric fields around curves. Two kinds of channel shapes were used for the examination. One (type I) has the same width in the curved region as in the straight region. The other (type II) is wider in the curved region than in the straight region. The difference in migration rates between long DNA and short DNA was larger in type II than in type I chips. We discuss the separation mechanism of the type II chip. (C) 2004 American Institute of Physics. [DOI: 10.1063/1.1776625]
\end{abstract}

\section{INTRODUCTION}

Separation of long deoxyribonucleic acid (DNA) by gel electrophoresis is still time consuming. ${ }^{1}$ Many technologies for the sizing of long DNA and related protocols have been proposed. $^{2}$ Especially, the recent development of microfabrication and nanofabrication techniques has provided a way to replace gel cross-linking with artificial obstacles to sieve molecules. ${ }^{3,4}$ However, for long DNA separation, even methods employing artificial obstacles need two-dimensional alternating electric fields. ${ }^{3}$ This fact implies that separation of long DNA by entanglement with cross-linked gel or artificial obstacles is not useful in a simple one-dimensional channel.

Using electrostatic interactions between DNA and the wall of a fused-silica capillary, Iki et al. demonstrated DNA separation without any separation media using capillary electrophoresis. ${ }^{5}$ Pernodet et al. performed DNA electrophoresis on a flat surface of silicon, and controlled the local friction between the adsorbed DNA and the surface by the surface energy of the modified silicon substrate. ${ }^{6}$ In both cases, the molecular interactions at the liquid-solid interface were regulated to increase the separation efficiency. On the other hand, Han and Craighead demonstrated entropy trapping in DNA electrophoresis without using a conventional gel system. ${ }^{7,8}$ The methods utilizing geometrical effects on long polymer conformation have greater potential for long DNA separation than sieving by entanglement with artificial obstacles. ${ }^{9}$

\footnotetext{
a) Author to whom correspondence should be addressed; Electronic mail: uedam@v006.vaio.ne.jp
}

In our previous papers, we investigated the curvature effect on long DNA molecules under electric fields ${ }^{9}$ and hydrodynamic flows. ${ }^{10}$ However, the curvature effect on molecules of different molecular weights under electric fields remains unclear. In the current paper, we investigate the curvature effect on long DNA and short fragmented DNA using two kinds of channels. One (type I) has the same width channel in the curved region as in the straight region and the other (type II) has a varying width channel in the curved region. In the following sections, we investigate the dynamics of long DNA through the curved regions in type I and type II chips.

\section{BACKGROUND}

We incorporate entropy gain based on a geometrical effect according to previous work. ${ }^{11,12}$ In order to introduce a long polymer into a straight capillary with a diameter $D$, we need the confinement energy $F_{\text {conf }}=T N(a / D)^{5 / 3}$, where $T$ is the absolute temperature (energy units), $a$ is the effective segment length, and $N$ is the segment number. This formula has a physical meaning under the geometrical condition $a$ $\ll D \ll R_{F} . R_{F}$ is the Flory radius of the real chain, which is calculated by $R_{F}=a N^{3 / 5}$.

Next, consider a polymer confined in a curved capillary, where $R$ is the average curvature radius of the wall between the inner and outer curvatures. In this case, the polymer confinement energy should be corrected in the following form: 
(a)

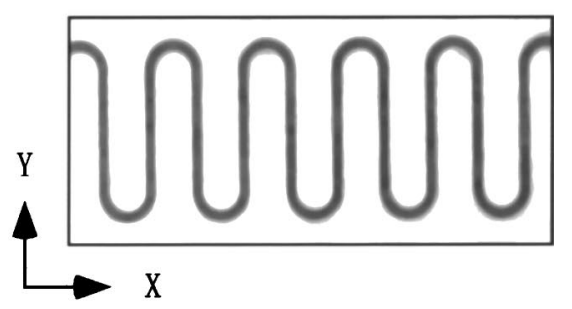

(b)

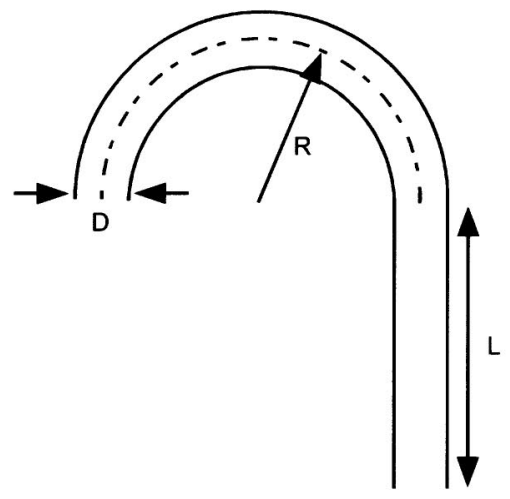

(c)

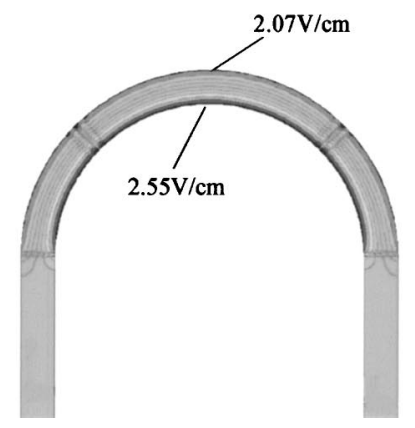

FIG. 1. (a) Photograph of a periodically curved channel chip (type I). $x$ and $y$ directions are defined as horizontal and vertical directions, respectively. (b) Schematic drawing of the capillary in the type I chip. The channel is $600 \mathrm{~nm}$ in width $(D), 480 \mathrm{~nm}$ in depth, with an average curvature radius of $3 \mu \mathrm{m}(R)$. The straight region is $15.7 \mu \mathrm{m}$ in length $(L)$. (c) Calculated electric field in the channel of the type I chip.

$$
F_{\text {conf }}=T N\left(\frac{a}{D}\right)^{5 / 3}-T \Delta \sigma[R]
$$

where $\Delta \sigma[R]$ is an entropy change induced by the curvature effect. For a polymer confined in the capillary with only one curvature radius, the entropy change is represented by $\Delta \sigma[R] \sim N a^{5 / 3} D^{1 / 3}(1 / R)^{2}$, where numerical factors are neglected (see the Appendix). Here, adding to the geometrical condition $a \ll D \ll R_{F}$, the size condition $l_{m}<L_{c}<\pi R$ should be satisfied, where $l_{m}$ is defined by $l_{m}$ $=2 \sqrt{(R+D / 2)^{2}-(R-D / 2)^{2}}=2 \sqrt{2 R D}$, and $L_{-} C$ is the average conformational size of DNA along the curved capillary.

Formula (1) means that the polymer entropy gain is restricted in a curved capillary. Therefore, when a polymer is confined in a straight capillary connected with curved regions as shown in Fig. 1(a), the polymer tends to spend more time in the curved regions than in the straight regions because of the entropy gain. ${ }^{10,12}$

Under weak hydrodynamic flows, long DNA satisfying the size condition $l_{m}<L_{-}<\pi R$ exhibits the curvature effect. ${ }^{10}$ However, under electric fields, the effect disappears due to the inhomogeneity of the electric field around the (a)

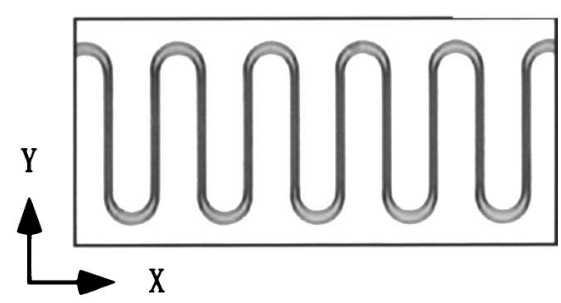

(b)

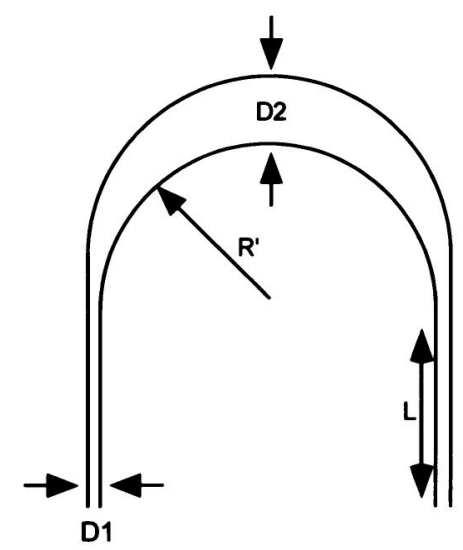

(c)

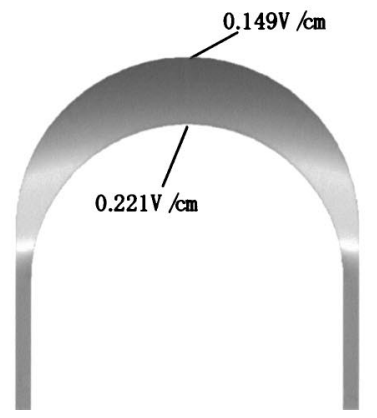

FIG. 2. (a) Photograph of the type II chip. The $x$ and $y$ directions are defined as the horizontal and vertical directions, respectively. (b) Schematic drawing of the capillary in the type II chip. In the straight regions, the channel is $0.35 \mu \mathrm{m}$ in width $(D 1)$ and $15.7 \mu \mathrm{m}$ in length $(L)$. The curved region has a varying channel width from $0.35 \mu \mathrm{m}(D 1)$ to $1.45 \mu \mathrm{m}(D 2)$. The curvature radius of the inner wall is $3 \mu \mathrm{m}\left(R^{\prime}\right)$. (c) Calculated electric field in the channel of the type II chip.

curve as shown in Fig. 1(c). ${ }^{9}$ Under an electric field, other factors such as electro-osmotic flow affect the DNA dynamics around a curve more than the entropic effect described by Eq. (1).

In order to enhance the geometrical effect, we increased the $D$ of the curved region, and decreased the $D$ of the straight region as shown in type II chip of Fig. 2(a). The confinement energy difference between the straight and the curved regions should be enlarged in the type II chip. Furthermore, the inhomogeneity of the electric field around the curved region is reduced and is localized at the interface between the straight and curved regions as shown in Fig. 2(c).

\section{EXPERIMENTAL DETAILS}

\section{A. Fabrication}

The nanocapillaries were fabricated on a quartz plate with a $2 \mathrm{~cm} \times 2 \mathrm{~cm}$ area by electron beam lithography, which was described in our previous work. ${ }^{9}$ 
Figure 1(a) shows a photograph of a periodically curved channel chip (type I), where the channel has the same width $(0.6 \mu \mathrm{m})$ and depth $(0.48 \mu \mathrm{m})$, both in the curved and straight regions. The curved channel is connected with wide straight channels (100 $\mu \mathrm{m}$ width) to reservoirs. As the arrows indicate, $x$ and $y$ directions are defined as horizontal and vertical directions, respectively. The curved region has an average curvature radius $R$ of $3 \mu \mathrm{m}$, and the straight region has a length $L$ of $15.7 \mu \mathrm{m}$ as shown in Fig. 1(b). Figure 1(c) shows the electric field in the type I chip channel calculated with ConventorWare (Conventor, Inc., USA) software. The dimensions used to calculate the field are as follows: capillary width is $0.6 \mu \mathrm{m}$, outer radius is $5.3 \mu \mathrm{m}$, inner radius is $4.7 \mu \mathrm{m}$, and the length of the straight region is $7.8 \mu \mathrm{m}$. The average field strength is $2.3 \mathrm{~V} / \mathrm{cm}$. The difference in field strength between the outer and inner wall around the curved region is calculated to be $0.48 \mathrm{~V} / \mathrm{cm}$.

Figure 2(a) shows a photograph of the type II chip. In the straight regions, the channel is $0.35 \mu \mathrm{m}$ in width $(D 1)$ and $15.7 \mu \mathrm{m}$ in length $(L)$. The curved region has a varying channel width from $0.35 \mu \mathrm{m}(D 1)$ to $1.45 \mu \mathrm{m}(D 2)$. The curvature radius of the inner wall is $3 \mu \mathrm{m}\left(R^{\prime}\right)$. Figure 2(c) shows the electric field in the type II chip channel calculated with ConventorWare software. The dimensions used to calculate the field are as follows: $D 1=0.3, D 2=1.3, R^{\prime}=3.0, L$ $=7.8 \mu \mathrm{m}$. The average field strength is $2.3 \mathrm{~V} / \mathrm{cm}$. The difference in field strength between the outer and inner wall around the curved region is calculated to be $0.07 \mathrm{~V} / \mathrm{cm}$.

\section{B. Materials and setup}

By means of fluorescence microscopy, T4 DNA (166 kbp, Nippon gene, Inc., Japan) and $\lambda$ DNA-HindIII digest [Marker I (23.13-0.13 kbp), Nippon gene, Inc., Japan] stained with YOYO-1 (Molecular Probes, Inc., Oregon) were visualized in $0.5 \times \mathrm{TBE}$ buffer $(45 \mathrm{mM}$ Trisborate, $1.25 \mathrm{mM}$ EDTA, $p \mathrm{H}=8.0$ ), which also includes $4 \%$ (v/v) 2-mercaptoethanol, $2.3 \mathrm{mg} / \mathrm{ml}$ glucose, $0.1 \mathrm{mg} / \mathrm{ml}$ glucose oxidase, $0.018 \mathrm{mg} / \mathrm{ml}$ catalase, and $0.3 \%$ polyvinylpyrrolidone (PVP; molecular weight $=10000$, TOKYOKASEIKOGYO CO., LTD., Japan). The Flory radius of T4 DNA in a free solution is estimated to be ca. $4.5 \mu \mathrm{m}\left(L_{\text {cont }}\right.$ $=0.34 \mathrm{~nm} / \mathrm{bp} \times 166 \mathrm{kbp}, a=100 \mathrm{~nm}, N=564)$. Therefore, in both type I and type II chips, the conformation of T4 DNA was deformed by the channel shape.

A silicon sheet ( $2 \mathrm{~mm}$ thickness), which has two holes (3 mm diameter) for buffer solution reservoirs, was placed on a chip cover plate. DNA-containing solutions $(1.5 \mu \mathrm{l})$ were dropped into the reservoirs. Then, the hole was sealed with another cover plate, which was connected to a microsyringe via a silicon tube. Prior to voltage application, the initial hydrodynamic flow in the channel was canceled by the regulation of reservoir pressure with the microsyringe. After that, constant voltages were applied with a power supply. Other details of the sample preparation are similar to those previously described. ${ }^{9}$
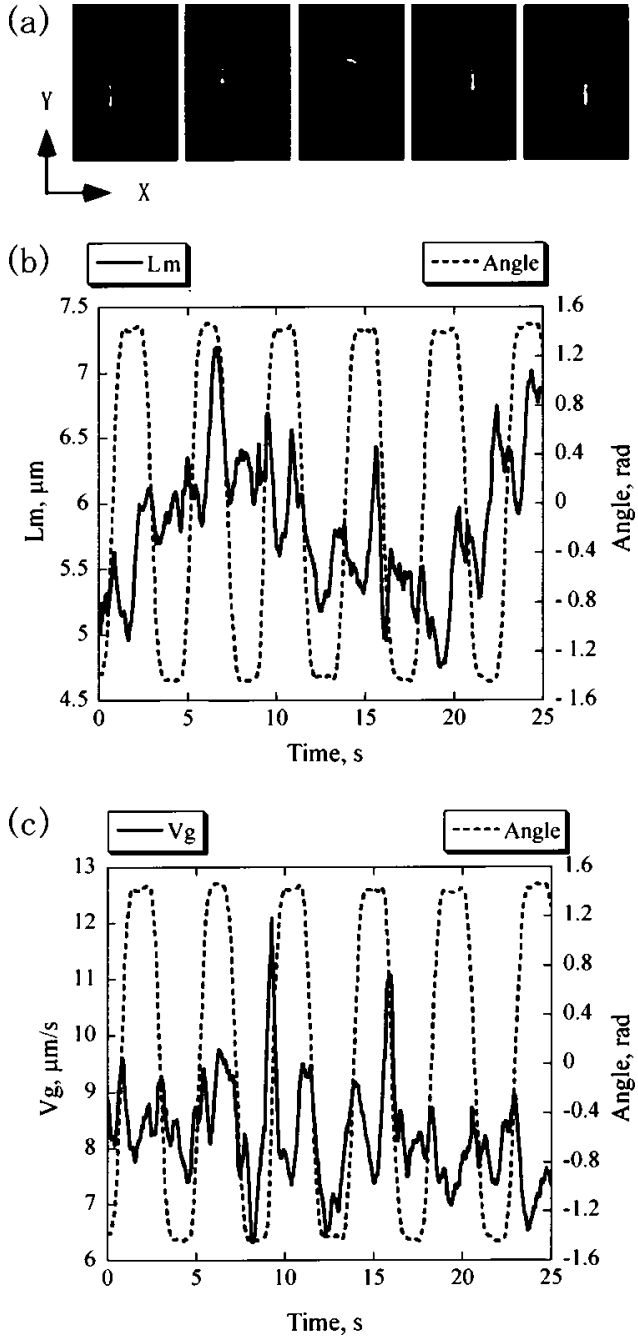

FIG. 3. (a) Fluorescence images of migrating T4 DNA under an electric potential of $1.0 \mathrm{~V}$ in the type I chip channel. Each image was taken at $0.5 \mathrm{sec}$ intervals. A scale of $10 \mu \mathrm{m}$ is indicated in the lower right. (b) Time courses in the maximum length ( $L_{m}$, solid line) of T4 DNA and the angle of $L_{m}$ to the $x$ direction (dotted line). (c) Time courses in the migration velocity ( $V_{g}$, solid line) of the center of mass of T4 DNA and the maximum length angle (dotted line).

\section{RESULTS}

\section{A. Data processing}

Figure 3(a) shows sequential fluorescence images of T4 DNA migrating in the type I chip. The images were taken at $0.5 \mathrm{sec}$ intervals from left to right. First, the fluorescence image (grayscale image) was translated into a black and white image (two value image). From the black and white image, the center of mass of DNA and the outline of the image were evaluated. Aftere that, the maximum length $L_{m}$, which is defined as the maximum length of crossing the outline, was calculated. Then, we calculated the angle of maximum length to the $x$ axis $(\theta,-\pi / 2<\theta<\pi / 2)$, and the migration velocity of the center of mass $\left(V_{g}\right)$ using the image processing software Cosmos32 (Library Inc., Tokyo, Japan). After taking the moving average, time courses of the maximum length $\left(L_{m}\right.$, solid line) and angle $(\theta$, dotted line), and the migration rate of the center of mass $\left(V_{g}\right.$, solid line) and angle ( $\theta$, dotted line) are shown in Fig. 3(b) and Fig. 3(c), respectively. 
In the plot of the maximum length angle $(\theta)$, the flat levels around \pm 1.5 rad indicate that the DNA was migrating in the straight region of the channel. On the other hand, monotonically increasing and decreasing regions in the angle change indicate that the DNA was migrating in the curved regions. Both in type I and type II chips, DNA molecules are stretched in the direction of the channel under electric fields. Therefore, we can use the maximum length angle as the indicator of the straight and curved regions. With the definition of the straight region of the channel as the region in which the angle is greater than $1.3 \mathrm{rad}$ (for T4 DNA) and $1.0 \mathrm{rad}$ (for short fragmented DNA), we calculated the averages of the maximum length $\left(L_{m s}\right.$ and $\left.L_{m c}\right)$ and the migration rate $\left(V_{g s}\right.$ and $\left.V_{g c}\right)$ in the straight and curved regions. The threshold angle distinguishing the straight region from the curved region depends on each DNA length. We evaluated 1.3 for T4 DNA and 1.0 for short fragmented DNA after investigating the test calculations for several molecules and the corresponding images in type I and II chips.

We measured individual molecules from $\lambda$ DNA-HindIII digest mixture as short fragment DNA. However, shorter DNA fragments than $6.5 \mathrm{kbp}$ were not counted because the size is comparable to noise level.

\section{B. Type I chip}

Figure 3(b) shows that T4 DNA is stretched periodically around curved regions in the type I chip. This stretching is accompanied by an increase in the migration rate $\left(V_{g}\right)$ as shown in Fig. 3(c). Because an electro-osmotic flow prevents the stretching of DNA (data not shown), we prevented the electro-osmotic flow by adding $0.3 \%$ PVP to the solution. In our previous work, we explained the stretching and acceleration of long DNA in curved channels due to inhomogeneity of electric fields. ${ }^{9}$ As shown in Fig. 1(c), the region around the inner wall of the curved channel has a higher electric field than the region near the outer wall. This means that the inhomogeneity of the electric field makes the effective width of the curved region smaller than that of the straight region. As a result, DNA was accelerated and stretched periodically around the curved region, as shown in Figs. 3(b) and 3(c).

Figure 4(a) shows the dependence of the conformational size of T4 DNA in the straight (open triangles) and curved regions (closed triangles) of the chip on the electric field. Under lower electric fields than $1 \mathrm{~V}$, DNA has a longer conformational size in curved regions than in straight regions. Under higher electric fields, the maximum length of T4 DNA is saturated to almost the same size both in straight and curved regions.

Figure 4(b) shows the dependence of the migration rates of T4 DNA and short fragmented DNA in the straight region on the electric field. We used DNA molecules with an $L_{m c}$ less than $3.5 \mu \mathrm{m}$ as short fragmented DNA. In the range of the electric field used, T4 DNA and fragmented DNA have the same migration rates. On the other hand, in curved regions, T4 DNA migrates slower than short fragmented DNA, as shown in Fig. 4(c). The difference in migration rates becomes larger as the electric field increases. Around curved regions, DNA migrates and adheres to the inner wall. ${ }^{9}$ There- (a)

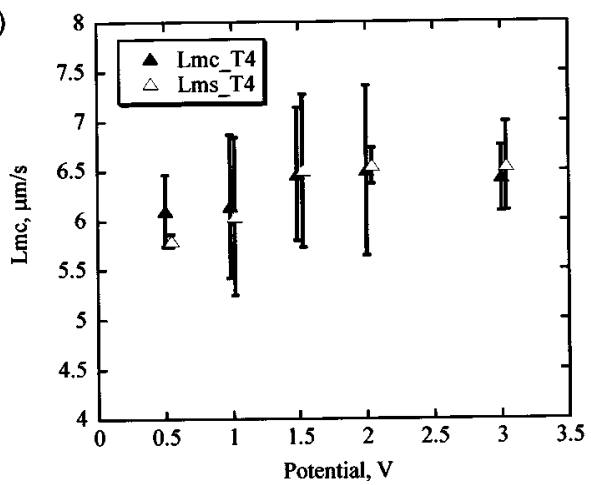

(b)

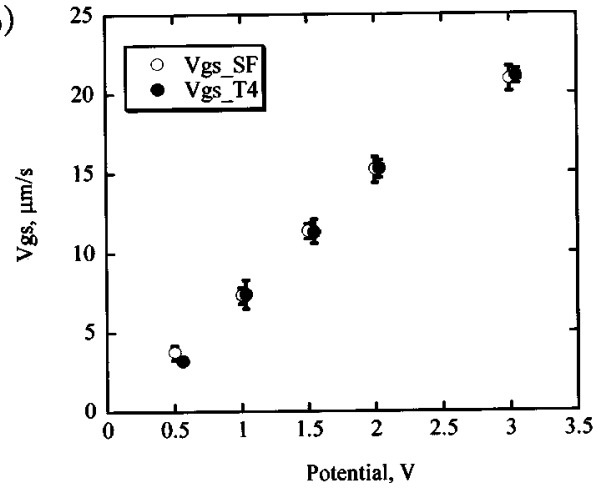

(c)

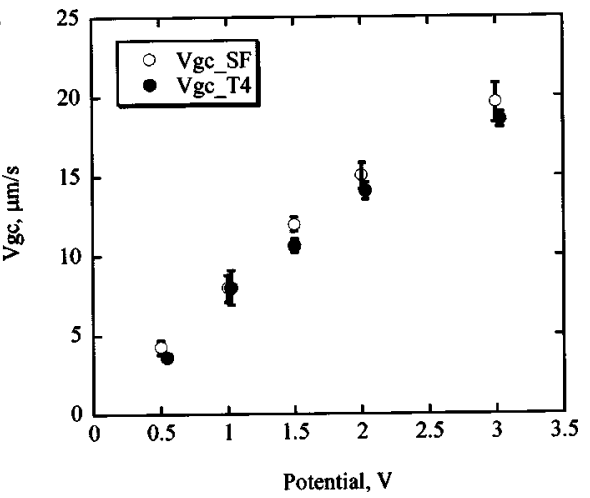

FIG. 4. (a) Plots of maximum length of T4 DNA in a type I chip vs potential. Open and closed triangles indicate maximum length in the straight region $\left(L_{m s}\right)$ and the curved region $\left(L_{m c}\right)$. (b) Plots of $V_{g}$ in straight regions vs potential. Open circles indicate migration rate of short fragments of DNA $\left(V_{g s_{S} S F}\right)$, closed circles indicate migration rate of T4 DNA $\left(V_{g s_{-} T 4}\right)$. (c) Plots of $V_{g}$ in curved regions vs potential. Open circles indicate the migration rate of short fragments of DNA $\left(V_{g c_{S} S F}\right)$, closed circles indicate the migration rate of T4 DNA $\left(V_{g c_{-} T 4}\right)$.

fore, it can be speculated that longer DNA interacts with the inner wall along longer distances and so migrates slower than short fragmented DNA. However, the differences of the migration rates are not very sensitive to the electric field strength.

\section{Type II chip}

In the type I chip, a mobility difference in the curved region depending on molecular length was generated, but the separation efficiency was not very high. In order to improve the separation efficiency, we investigated the behavior of the type II chip.

Figure 5(a) shows sequential fluorescence images of T4 DNA under an electric field of $1.0 \mathrm{~V}$ in the type II chip. Each 
(a)
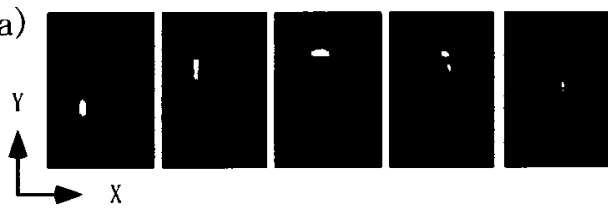

(b)

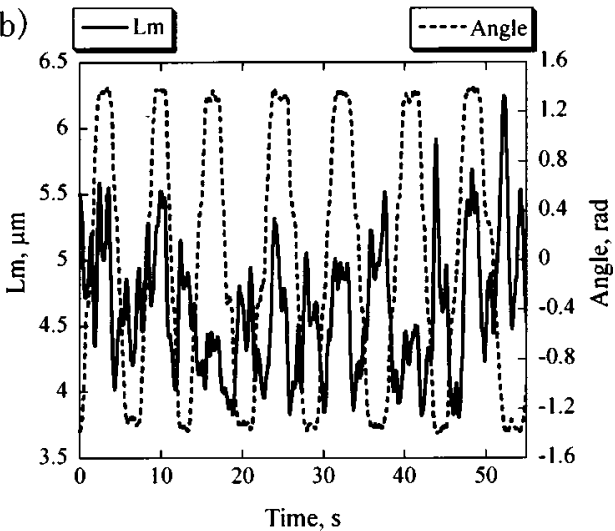

(C)

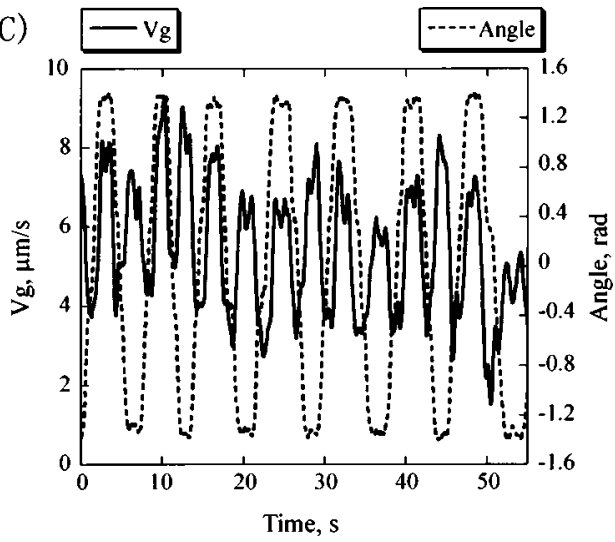

FIG. 5. (a) Fluorescence images of migrating T4 DNA under an electric potential of $1.0 \mathrm{~V}$ in the type II chip channel. Each image was taken at $1 \mathrm{sec}$ intervals. (b) Time courses in the maximum length $\left(L_{m}\right.$, solid line) of T4 DNA and the angle (dotted line) of $L_{m}$ to the $x$ direction. (c) Time courses in the migration velocity $\left(V_{g}\right.$, solid line) of the center of mass of T4 DNA and the angle of the maximum length to the $x$ axis (dotted line).

image was taken at $1 \mathrm{sec}$ intervals from left to right. Time courses of $L_{m}$ and $\theta, V_{g}$ and $\theta$ are shown in Figs. 5(b) and 5 (c), respectively. DNA is stretched and accelerated in the straight region, and is released and decelerated in the curved region periodically. This tendency is completely opposite to the behavior observed in the type I chip. Furthermore, the changes of $L_{m}$ and $V_{g}$ in the straight regions of the type II chip exhibit a double peak wave form. The greater width in the curved region of the type II chip reduces the electric field inhomogeneity in the normal direction, as shown in Fig. 2(c). The electric field inhomogeneity is localized at the interfaces between the straight and curved regions.

Figure 6(a) shows plots of the maximum length of T4 DNA in the type II chip versus potential. Open and closed triangles indicate the maximum length in the straight $\left(L_{m s_{T 4}}\right)$ and the curved $\left(L_{m c} T 4\right)$ regions, respectively. Both in the straight and curved regions, stretching of the T4 DNA varies directly with the increase in potential. The tendency is not saturated in this potential range.

Figure 6(b) shows plots of $V_{g}$ in straight and curved regions versus potential. Open and closed circles indicate the (a)

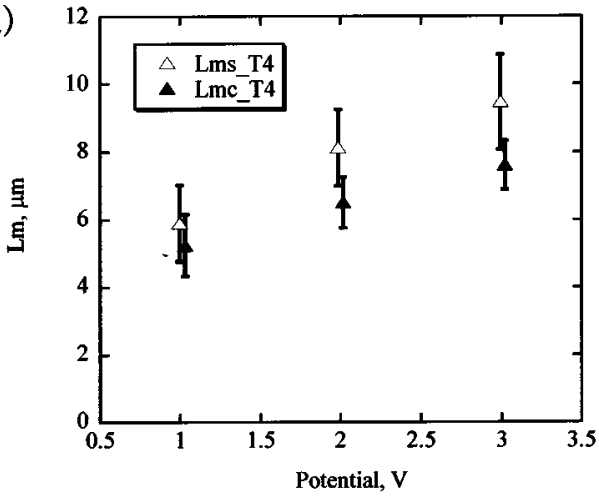

(b)
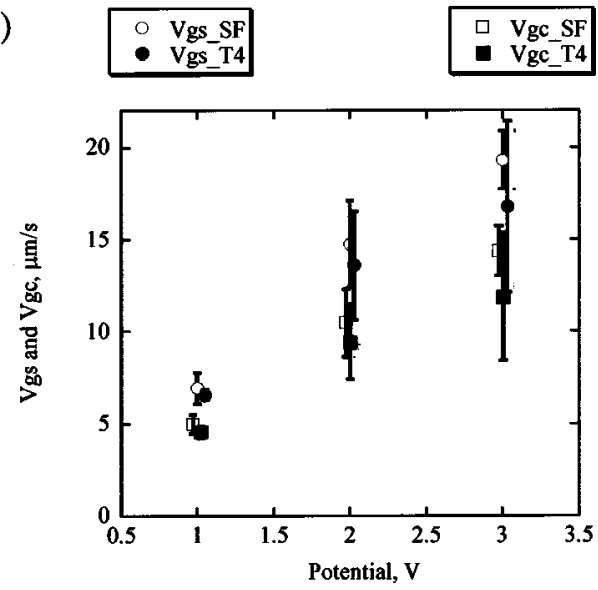

FIG. 6. (a) Plots of maximum length of T4 DNA in the type II chip vs potential. Open and closed triangles indicate the maximum length in the straight $\left(L_{m s_{T} T 4}\right)$ and the curved $\left(L_{m c}{ }_{T 4}\right)$ regions, respectively. (b) Plots of $V_{g}$ in straight and curved regions vs potential. Open circles indicate the migration rate of short fragments of DNA in the straight region $\left(V_{g s} S F\right)$, closed circles indicate the migration rate of T4 DNA in the straight region $\left(V_{g s_{-} T 4}\right)$. Open triangles indicate the migration rate of short fragments of DNA $\left(V_{g c_{-} S F}\right)$, closed triangles indicate the migration rate of T4 DNA $\left(V_{g c_{-} T 4}\right)$.

migration rates of short fragments of DNA $\left(V_{g S_{-} S F}\right)$ and T4 DNA $\left(V_{g s_{-} T 4}\right)$ in the straight region, respectively. Open and closed rectangles indicate the migration rate of short fragments of DNA $\left(V_{g c_{-} S F}\right)$ and T4 DNA $\left(V_{g c_{-} T 4}\right)$ in curved regions, respectively. Both in the straight and curved regions, the short fragmented DNA migrates faster than T4 DNA. The differences between $V_{g s_{-} S F}$ and $V_{g s_{-} T 4}$, and between $V_{g c_{-} S F}$ and $V_{g c_{-} T 4}$ still increase monotonically with the increase in potential.

\section{DISCUSSION}

In this section, we discuss three factors regarding DNA separation in the type II chip: local friction between DNA and the capillary wall, the process of entrance into the curved region, and the nonuniform electric field. Although they are connected with each other, we discuss each separately.

\section{A. Local friction between DNA and capillary wall}

We assumed that our capillary wall is a hard repulsive wall in the derivation of Eq. (A3) (Appendix). That is correct as a first approximation, because in our experiments, PVP, which prevents electro-osmotic flow and reduces the interaction between DNA and the capillary wall, was added to the 
(a)
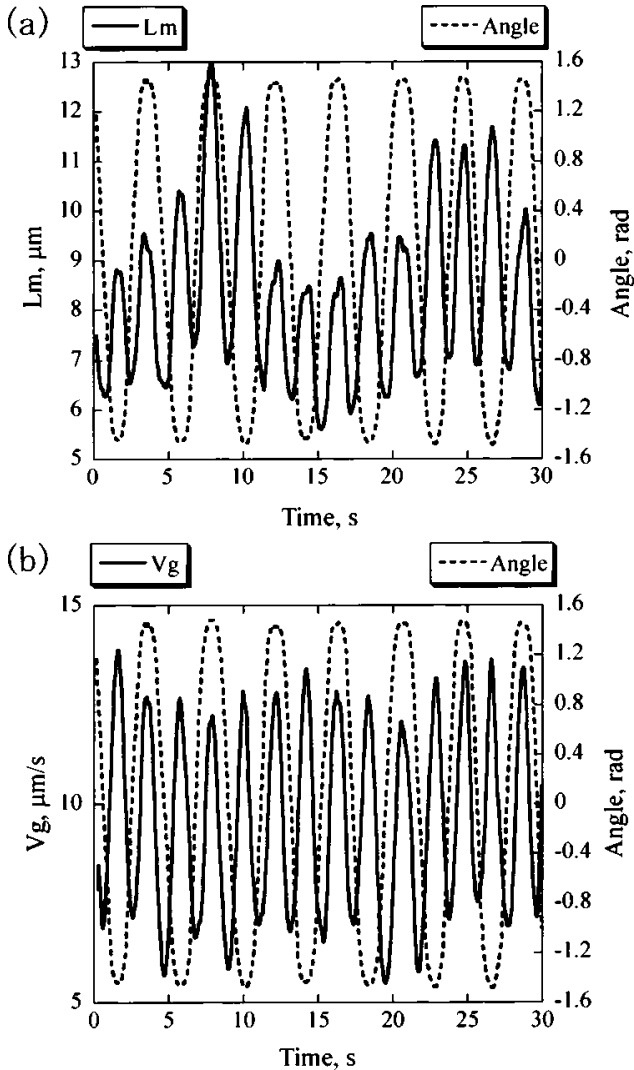

FIG. 7. (a) Time courses of the maximum length $\left(L_{m}\right)$ of T4 DNA and the angle of $L_{m}$ to the $x$ direction under a potential of $3 \mathrm{~V}$ in the type II chip. (b) Time courses in the migration velocity $\left(V_{g}\right)$ of the center of mass of T4 DNA and the angle of the maximum length to the $x$ axis under a potential of $3 \mathrm{~V}$ in the type II chip.

solutions. ${ }^{13,14}$ However, comparing Fig. 6(b) to Fig. 4(b), even in the straight region, the migration velocity is dependent on molecular size due to the narrower capillary. This implies that the local friction between DNA and the wall may be occurring. ${ }^{6}$ In order to check this point, we should investigate the electrophoresis of long DNA in long straight narrow (or shallow) capillaries in our future work.

\section{B. Entrance process from a wider channel to a narrower channel}

As mentioned above, Figs. 5(b) and 5(c) show double peaks in the changes in $L_{m}$ and $V_{g}$ under an electric potential of $1 \mathrm{~V}$. This means that the migration time in the straight region is longer than the relaxation time of the stretched DNA. The first peak indicates the process of entrance into the narrow straight channel from the wider curved region.

However, under higher potentials, both $L_{m}$ and $V_{g}$ exhibit a single peak wave form as shown in Fig. 7(a) and 7(b). Under these conditions, DNA migrates up to the next curve before completing the relaxation. Therefore, under higher electric fields, the entrance process into the narrow channel dominates the migration process in the straight region. This means that the entrance process is one of the causes of molecular sieving.

In order to provide insight into the entrance dynamics of DNA in the type II chip, we compared the entrance process of T4 DNA under electric potentials of $1 \mathrm{~V}$ and $3 \mathrm{~V}$ as
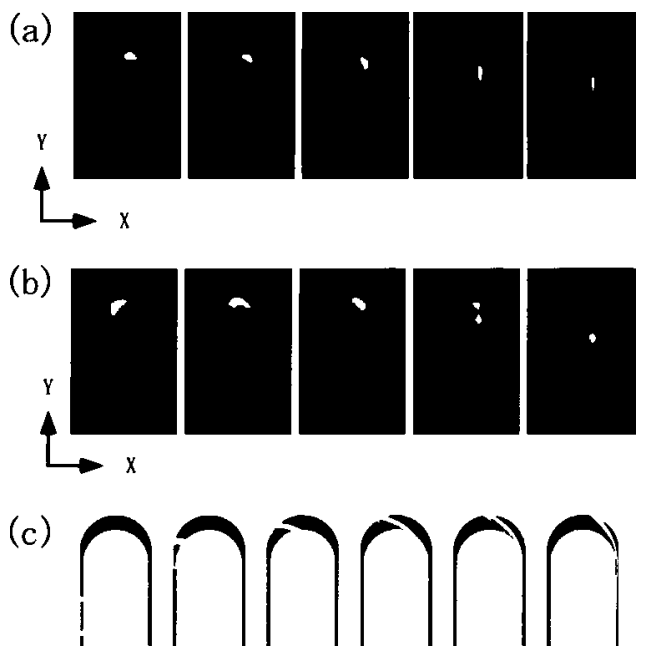

FIG. 8. Entrance process of T4 DNA into a narrow straight channel. Fluorescence images were taken from left to right at $0.33 \mathrm{sec}$ interval. (a) Under $1 \mathrm{~V}$ in a type II chip. (b) Under $3 \mathrm{~V}$ in a type II chip. (c) Calculated entrance process of a sample plug into a narrow channel in a type II chip by ConventorWare. Each frame was taken from left to right at $1 \mathrm{sec}$ intervals.

shown in Figs. 8(a) and 8(b), respectively. Each image was taken at $0.33 \mathrm{sec}$ intervals from left to right. Under a potential of $1 \mathrm{~V}$, DNA enters almost entirely at once. Faster migrating sections extending into the straight channel from entangled DNA are rarely observed. On the other hand, under the $3 \mathrm{~V}$ potential, DNA enters from the leading end in order (head first or tail first), as shown in Fig. 8(b). Although the main tangled parts tend to stay in the curved region, unraveling occurs with the migration of the leading end. However, in this channel size $(0.35 \mu \mathrm{m}$ width), small entanglements enter before being completely released, as shown in Fig. 8(b). For further improvement of separation quality, we should use a narrower capillary. ${ }^{15}$ Taking into account the inflexibility of double-stranded DNA (dsDNA) (the persistence length of dsDNA is about $50 \mathrm{~nm}),{ }^{16}$ it may be preferable to have the width of the narrow capillary less than $100 \mathrm{~nm}$.

\section{Nonuniform electric field}

Figure 8(c) shows sequential images of the sample plug migrating in a type II chip (the average electric field is $2.3 \mathrm{~V} / \mathrm{cm}$ ) calculated by ConventorWare (Conventor, Inc., USA) software. The sample plug is "monomer gas" made of point charges with a mobility of $2.5 \times 10^{-4} \mathrm{~cm}^{2} \mathrm{~V} \mathrm{~s}$, and a concentration of $0.13 \mu \mathrm{mol} / \mathrm{l}$. As shown in Fig. 8(c), the sample plug is stretched during the migration along the curved channel because of the nonuniform electric field. ${ }^{17,18}$ However, in the case of DNA, the stretching in the type II chip is observed during the entrance process into a straight region, not while moving through the curved part. The reason for this is the fact that DNA segments are connected with each other. Therefore, the entropic elasticity of DNA plays an important role in its dynamics around the curved channel as happens in the type II chip. However, the head-first entrance under $3 \mathrm{~V}$, as shown in Fig. 8(b), may be induced by DNA stretching with the nonuniform electric field. 
Han et al. reported that longer DNA has a larger mobility in electrophoresis using an entropy-trapping array. ${ }^{7,8}$ Tessier et al. studied the separation mechanism of long DNA in the entropy-trapping array by means of Monte Carlo simulation, and investigated the entrance process of long DNA from a deep region to a shallow region. ${ }^{19}$ They suggested that a critical "hernia nucleation" (see Ref. 19) dominates the entrance process of long DNA, and the head-first or tail-first entrance dominates the entrance of shorter DNA. However, in our case, longer DNA has a smaller mobility, as shown in Fig. 6(b), and the head-first or tail-first entrance dominates the entrance process of even long DNA as shown in Fig. 8(b). We assume that the differences between our results and the results of Han et al. arise from the differences in the DNA entrance process (hernia nucleation or head-first entrance) in each chip design. Further investigation using experimental and computational approaches is required to resolve these discrepancies.

\section{CONCLUSION}

We investigated the curvature effect on long polymer dynamics using microcapillary and nanocapillary devices. Long DNA exhibits large conformational changes in the curved channels because of the inhomogeneity of the electric fields around curves. Two kinds of channel shapes were used for the examination. One (type I) has the same width in the curved region as in the straight region. The other (type II) is wider in the curved region than in the straight region. The differences in migration rates between long DNA and short DNA were larger in the type II than in the type I channel. In the type II chip, molecular sieving occurs during the entrance process of DNA from the curved region to the narrow straight region, and from the straight region to the curved region. Using a narrower straight region will improve the separation quality.

\section{ACKNOWLEDGMENTS}

This work was partially supported by a grant from the Core Research for Evolutional Science and Technology (CREST) from the Japan Science and Technology Corporation (JST). The authors gratefully acknowledge helpful discussions with Dr. Y. Y. Suzuki (NTT Basic Research Laboratories) and Dr. S. Miyamoto (Marubeni Solutions Corp.) on several aspects of this work.

\section{APPENDIX}

Suppose a geometrical neutral surface (abbreviated $n$ surface) which is equidistant from two walls confining a polymer, and introduce a local orthogonal coordinate system $x=(u, v, w)$, as shown in Fig. 9(a). The surface coordinates $u$, $v$ correspond to principal directions of the $n$ surface with the principal curvatures $\kappa_{u}=1 / R_{u}$ and $\kappa_{v}=1 / R_{v}$. The width direction $w$ is normal to the $n$ surface. Two principal directions are orthogonal, and the curvature of an arbitrary direction associated with an azimuthal angle $\theta$ in Fig. 10(a) is given by $\kappa(\theta)=\cos ^{2} \theta / R_{u}+\sin ^{2} \theta / R_{v}$. The angular average over $\theta$ of the curvature $\kappa(\theta)$ at the point $P_{0}$ is simply the mean curvature $H=\left(R_{u}^{-1}+R_{v}^{-1}\right) / 2$. (a)

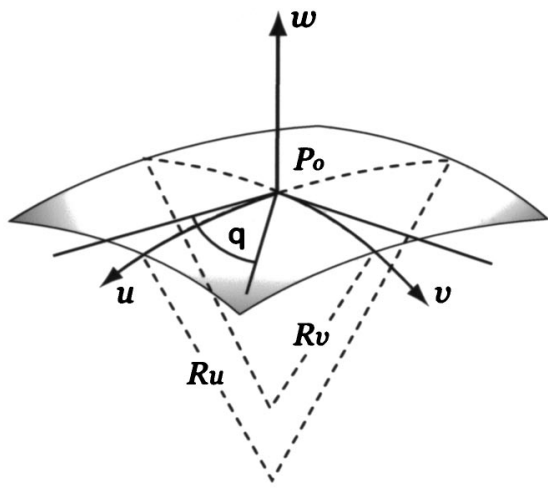

(b)

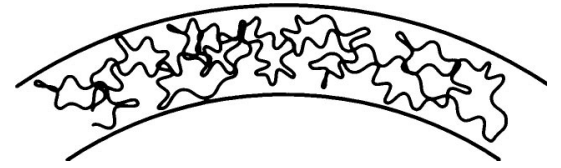

FIG. 9. (a) Local orthogonal coordinate system $\mathbf{x}=(u, v, w) . u$ and $v$ correspond to the principal directions which are tangent to the neutral surface at a point $P_{0}=(0,0,0)$ with the principal curvatures $\kappa_{u}$ and $\kappa_{v}$. The principal radii are given by $R_{u}=1 / \kappa_{u}$ and $R_{v}=1 / \kappa_{v}$. A curvature corresponding to an arbitrary direction with an azimuthal angle $\theta$ is $\kappa(\theta)=\cos ^{2} \theta / R_{u}+\sin ^{2} \theta / R_{u}$. (b) Long flexible polymer confined in a curved box.

Using $R_{u}$ and $R_{v}$, we can formulate the entropy gain of a polymer confined between two parallel curved walls with distance $D\left(D \ll R_{u}, R_{v}\right)$ as shown in Fig. 9(b).

Segments touching the walls should recoil because of the repulsive hard interaction. Let $z$ be the number of the nearest position for the segment. At exactly flat surfaces, $z$ is halved. The curvature effect could be induced by the deviation from $z / 2$. Hereafter, we single out a configuration in which the polymer with $N$ segments touches the walls every $N^{\prime}$ segment. The sum of the number of collisions with (and recoils from) the outer and inner walls ( $M_{+}$and $M_{-}$, respectively) is $M_{+}+M_{-} \sim N / N^{\prime}$. Then the number $W$ of total configurations of the confined polymer may be represented by

$$
W \sim z^{N}\left(\frac{1}{2}\right)^{N / N^{\prime}}\left(1-2 f_{+}\right)^{M_{+}}\left(1+2 f_{-}\right)^{M_{-}},
$$

where $f_{+}$is the fraction of the deficit in the number of configurations due to collisions with the outer wall [see Fig.

(a)

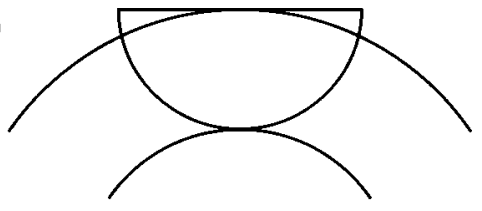

(b)

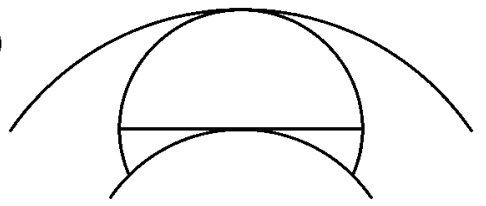

FIG. 10. Two-dimensional analog of angular geometrical constraints: Hatched regions correspond to (a) deficit of configurations on the outer wall and (b) surplus of configurations on the inner wall. Notice that the deficit is less than the surplus. 
10(a)] and $f_{-}$is the fraction of the surplus in the number of configurations due to collisions with the inner wall [see Fig. 10(b)]. We have omitted numerical constants and a combination factor and ignored the variations of $M_{+}$and $M_{-}$.

The entropy is calculated as

$$
S=\ln W \sim \sigma_{0}+\sigma_{1}+\Delta \sigma .
$$

The first term $\sigma_{0}=N \ln z$ is the entropy of a polymer in a free space. The second term $\sigma_{1} \sim-\left(N / N^{\prime}\right)$ is the entropy loss between planer walls.

The last term $\Delta \sigma$ is the entropy gain due to the curvatures compared with the exactly flat planes. We have

$$
\Delta \sigma=-2 M_{+} f_{+}+2 M_{-} f_{-},
$$

where $f_{+}$and $f_{-}$are assumed to be small values. Eq. (A2) means that the total entropy change is the sum of the number $\left(M_{+}\right.$and $\left.M_{-}\right)$of collisions times entropy change $\left(f_{+}\right.$and $\left.f_{-}\right)$ per collision.

Now we will estimate the deficit $f_{+}$and the surplus $f_{-}$, treating polymers as blobs. When principal radii $R_{u}$ and $R_{v}$ are large compared to $D$, the fractions of the deficit and the surplus of solid angles being available for blobs can be calculated using the following equation:

$$
\frac{1}{4 \pi} \int_{0}^{2 \pi} \frac{D}{2} \kappa(\theta) d \theta=\frac{D}{4} \frac{1}{2}\left(\frac{1}{R_{u}}+\frac{1}{R_{v}}\right) .
$$

Thus the fractions are written as

$$
f_{+}=\frac{D}{4} H\left(\frac{D}{2}\right), \quad f_{-}=\frac{D}{4} H\left(\frac{-D}{2}\right),
$$

with

$$
H(w)=\frac{1}{2}\left(\frac{1}{R_{u}+w}+\frac{1}{R_{v}+w}\right),
$$

where $H(D / 2)$ and $H(-D / 2)$ are the mean curvatures of the outer and inner walls, respectively. Notice that $f_{-}>f_{+}$for $R_{u}>0$ and $R_{v}>0$.

The numbers of collisions with the two curved surfaces are not equal and they are modified by two factors. The first factor depends on surface area change. We represent this factor with $S(w) / S(0)$, where $S(w)=\left(R_{u}+w\right)\left(R_{v}+w\right)$.

The second factor depends on local density profile changes. The density changes are induced because of the local entropy gain or loss. Here we assume that the number of collisions has a linear dependence on the ratio of the total angular configurations of our blobs at points on the walls. We therefore multiply a factor $\left(1-2 f_{+}\right)$or $\left(1+2 f_{-}\right)$to the average number of collisions $N / 2 N^{\prime}$.

Thus we write

$$
\begin{aligned}
& M_{+} \sim \frac{N}{2 N^{\prime}} \frac{S(D / 2)}{S(0)}\left(1-2 f_{+}\right), \\
& M_{-} \sim \frac{N}{2 N^{\prime}} \frac{S(-D / 2)}{S(0)}\left(1+2 f_{-}\right),
\end{aligned}
$$

which hold $M_{+}+M_{-} \sim N / N^{\prime}$.

From Eq. (A2), brief calculation yields

$$
\Delta \sigma \sim N \zeta\left(\frac{1}{R_{u}}-\frac{1}{R_{v}}\right)^{2},
$$

where $\zeta=D^{2-1 / \nu} a^{1 / \nu}$. For self-avoiding chains, we have $\zeta$ $=a^{5 / 3} D^{1 / 3}$.

In the special case of Eq. (A3), we have $\Delta \sigma$ $\sim N a^{5 / 3} D^{1 / 3}(1 / R)^{2}$ for a self-avoiding chain confined between cylindrical shells $\left(R_{u}=R, R_{v}=\infty\right)$. Here, we can regard the generic formula as the curvature entropy gain of a selfavoiding chain confined in one-dimensional curved capillary as well.

${ }^{1}$ S. Gurrieri, S. B. Smith, and C. Bustamante, Proc. Natl. Acad. Sci. U.S.A. 96, 453 (1999).

${ }^{2}$ J. Jing et al., Proc. Natl. Acad. Sci. U.S.A. 95, 8046 (1998).

${ }^{3}$ O. Bakajin, T. A. Duke, J. Tegenfeldt, C. F. Chou, S. S. Chan, R. H. Austin, and E. C. Cox, Anal. Chem. 73, 6053 (2001).

${ }^{4}$ L. R. Huang, J. O. Tegenfeldt, J. J. Kraeft, J. C. Sturm, R. H. Austin, and E. C. Cox, Nat. Biotechnol. 20, 1048 (2002).

${ }^{5}$ N. Iki, Y. Kim, and E. S. Yeung, Anal. Chem. 68, 4321 (1996).

${ }^{6}$ N. Pernodet, V. Samuilov, K. Shin, J. Sokolov, M. H. Rafailovich, D. Gersappe, and B. Chu, Phys. Rev. Lett. 85, 5651 (2000).

${ }^{7}$ J. Han and H. G. Craighead, Science 288, 1026 (2000).

${ }^{8}$ J. Han and H. G. Craighead, Anal. Chem. 74, 394 (2002).

${ }^{9}$ M. Ueda, T. Hayama, Y. Takamura, Y. Horiike, and Y. Baba, Electrophoresis 23, 2635 (2002).

${ }^{10}$ M. Ueda, T. Hayama, Y. Takamura, Y. Horiike, T. Dotera, and Y. Baba, Jpn. J. Appl. Phys., Part 1 43, 1649 (2004).

${ }^{11}$ K. Yaman, P. Pincus, F. Solis, and T. A. Witten, Macromolecules 30, 1173 (1997).

${ }^{12}$ T. Dotera and Y. Y. Suzuki, Phys. Rev. E 62, 5318 (2000).

${ }^{13}$ Q. Gao and E. S. Yeung, Anal. Chem. 70, 1382 (1998).

${ }^{14}$ S. Ho Kang, M. R. Shortreed, and E. S. Yeung, Anal. Chem. 73, 1091 (2001).

${ }^{15}$ M. Foquet, J. Korlach, W. Zipfel, W. W. Webb, and H. G. Craighead, Anal. Chem. 74, 1415 (2002).

${ }^{16}$ C. Rivetti, C. Walker, and C. Bustamante, J. Mol. Biol. 280, 41 (1998).

${ }^{17}$ M. J. Chacron and G. W. Slater, Phys. Rev. E 56, 3446 (1997).

${ }^{18}$ L. L. Frumin, S. E. Peltek, and G. V. Zilberstein, Phys. Rev. E 64, 021902 (2001).

${ }^{19}$ F. Tessier, J. Labrie, and G. W. Slater, Macromolecules 35, 4791 (2002). 